\title{
Dietary Calcium or Vitamin D Intake and Type 2 Diabetes Mellitus Incidence: A Dose-Response Meta-analysis
}

\author{
Lingling $\mathrm{Li}^{1, \dagger}$, Qianwen $\mathrm{Li}^{1, \dagger}$, Junhao Wang ${ }^{1}$, Hao Wang ${ }^{1}$, Yongjuan Xin ${ }^{1}$, Shenshen Zhang ${ }^{1}$, \\ Ying $\mathrm{Cao}^{2}$, Mengyao Zhou ${ }^{1}$, Wenjie Yang, ${ }^{1,}$ \\ ${ }^{1}$ Precision Nutrition Innovation Center, Department of Nutrition, College of Public Health, Zhengzhou University, Zhengzhou, China \\ ${ }^{2}$ School of Education and Child Development, Drury University, Springfield, Missouri, USA
}

\section{Email address:}

linglingli1030@163.com (Lingling Li), lqw9319@163.com (Qianwen Li), kevinwang93@163.com (Junhao Wang), wanghao2016@zju.edu.cn (Hao Wang),yjxinzzu@163.com (Yongjuan Xin), zsslb2005@163.com (Shenshen Zhang), 18844504070@163.com (Mengyao Zhou),ywjie@zzu.edu.cn (Wenjie Yang), ycao@drury.edu (Ying Cao)

*Corresponding author

$\dagger$ Lingling Li and Qianwen Li are co-first authors.

\section{To cite this article:}

Lingling Li, Qianwen Li, Junhao Wang, Hao Wang, Yongjuan Xin, Shenshen Zhang, Ying Cao, Mengyao Zhou, Wenjie Yang. Dietary Calcium or Vitamin D Intake and Type 2 Diabetes Mellitus Incidence: A Dose-Response Meta-analysis. International Journal of Diabetes and Endocrinology. Vol. 6, No. 1, 2021, pp. 36-45. doi: 10.11648/j.ijde.20210601.17

Received: February 2, 2021; Accepted: February 15, 2021; Published: March 4, 2021

\begin{abstract}
Background: Dietary Calcium and vitamin D intake may have effects on the onset risk of type 2 diabetes mellitus (T2DM), whereas the results of existing studies are inconsistent. We performed a meta-analysis of prospective studies to evaluate the association between dietary calcium or vitamin D intake and the onset risk of T2DM. Methods: We searched PubMed, Web of Science, and Embase for relevant studies published through 5 Jan 2021. The relative risks (RRs) with $95 \%$ confidence intervals (CIs) of T2DM in relation to dietary calcium or vitamin D intake were pooled with a fix or random-effects model. Results: 22 cohort studies were included in this meta-analysis, which comprising 31027 cases and 448578 participants. We found dietary calcium (RR: 0.84 ; 95\% CI: $0.76-0.93$ ) rather than dietary vitamin D (RR: $1.00 ; 95 \%$ CI: $0.92-1.08$ ) reduced the onset risk of T2DM. Subgroup analysis showed the efficacy of dietary calcium intake is only in Asian, women and older population. Dose-response analysis revealed that each $300 \mathrm{mg} /$ day increase in dietary calcium intake reduces the risk of T2DM by $8 \%$. However, the increasing dietary vitamin D intake does not affect the risk of T2DM. Conclusions: Dietary calcium intake reduces the onset risk of T2DM but dietary vitamin D has no effect.
\end{abstract}

Keywords: Calcium, Vitamin D, Type 2 Diabetes Mellitus, Meta-analysis

\section{Introduction}

Diabetes is one of the most common global chronic diseases with a staggering 463 million cases in 2019 , and expected to be 578 million by 2030,700 million by 2045 [1]. Several large randomized controlled trials (RCTs) demonstrated that dietary interventions could minimize the onset risk of T2DM $[2,3]$, which could ease the burden of this disease worldwide.

$\mathrm{Ca}^{2+}$ is the most abundant ion in the body, which plays both a structural and functional role in bones [4]. Except for its well-known function, it was found that insulin secretion is a calcium-dependent process [5] and also calcium is vital for insulin-mediated intracellular processes in tissues that responding to insulin, such as muscle and fat [6]. Vitamin D is essential for optimal musculoskeletal health because it promotes calcium absorption, mineralization of osteoid tissue formation in bone, and maintenance of muscle function [7]. Its deficiency has been linked to the onset of T2DM mediated by insulin secretion, insulin sensitivity, and systemic inflammation [8].

Previous reviews have summarized the evidence linking dietary calcium or vitamin $\mathrm{D}$ intake with the development of T2DM. However, these reviews were limited by study design, number of participants, and heterogeneity among studies [9, 10]. Here, we conducted a meta-analysis of prospective studies to quantify the effects of dietary vitamin D and 
calcium intake on T2DM incidence risk in non-diabetic subjects, as well as to explore the potential of heterogeneity.

\section{Materials and Methods}

This meta-analysis was conducted according to the Meta-analysis of Observational Studies in Epidemiology (MOOSE) protocol [11]. We registered our study protocol with the International Prospective Register of Systematic Reviews, PROSPERO (registration no: CRD42020182946).

Search strategy. Eligible studies were searched by two investigators (authors L.L. and Q.L.) in PubMed, Web of Science, and Embase from their inception to 5 Jan 2021, using the following search terms: $(((()(((($ calcium [Title/Abstract]) OR (dairy products [Title/Abstract])) OR (dairy [Title/Abstract])) OR (milk [Title/Abstract])) OR (cheese [Title/Abstract])) OR (yogurt [Title/Abstract])) OR (cream [Title/Abstract])) OR (vitamin D [Title/Abstract])) AND (diabetes [Title/Abstract])) AND ((( prospective) OR (follow-up)) OR (cohort)) OR (longitudinal)). In addition, the reference list of the identified articles and relevant reviews and meta-analyses were retrieved manually for additional potential publications.

\subsection{Inclusion and Exclusion Criteria}

Eligible studies were selected on the basis of following criteria: (1) cohort studies; (2) study subjects were non-diabetic individuals, including those with normal blood glucose and prediabetes at baseline; (3) the exposure was the intake of dietary calcium and/or vitamin D; (4) the outcome was T2DM; (5) the relative risk (RR) or hazard ratio (HR), with the corresponding $95 \%$ confidence interval $(95 \% \mathrm{CI})$ for the risk of T2DM were reported or could be calculated; (6) for the dose-response analysis, the intake of dietary calcium or vitamin D in each category was either reported or could be calculated; (7) the study was published in English. If a study reported several RRs (HRs), the most adjusted ratio for the highest level versus the lowest level was selected to calculate the pooled RR. If the same data were reported in more than one article, we selected the article including the most detailed data. We excluded publications if they were abstracts, meta-analyses, reviews, case-reports, articles without full text, studies involving non-human species, and articles lacking of essential data.

\subsection{Data Extraction and Quality Assessment}

Data extraction was performed independently by two investigators (authors L.L. and Q.L.): The name of the first author, year of publication, country, geographic location, age, gender, sample size (number of participants and cases), years of follow-up, intake comparison, the corresponding fully adjusted RR (or HR) with 95\% CIs and adjusted covariates. The quality of each cohort study was also independently evaluated by investigators mentioned above according to the Newcastle-Ottawa Scale, with a maximum of nine points [12]. Inconsistencies were resolved through group discussions to reach consensus.

\subsection{Statistical Analysis}

Initially, If $I^{2}>50 \%$, the DerSimonian and Laird random-effects model was used; otherwise, the Mantel-Haenszel fixed-effects model was used [13]. The pooled estimates were calculated using the RRs and 95\% CIs for all studies. We assumed that HRs reported in the publications were approximately RRs. Any results stratified by gender were treated as independent reports.

Heterogeneity among studies was evaluated by $Q$ test and $I^{2}$ statistic. The cut-off points were $25 \%, 50 \%$, and $75 \%$ representing low, moderate, and high degrees of heterogeneity, respectively [14]. To find the probable sources of heterogeneity, meta-regression and subgroup analyses were both performed to evaluate the effect of modifying potential key covariates [15]. Subgroup analyses were performed to assess the potential modifying effects of the following variables on outcomes: geographic location, gender, mean age, follow-up years, baseline characteristics of participants, and adjustment by age, energy, magnesium, calcium, vitamin D or alcohol intake.

A "leave-one-out" sensitivity analysis was used to evaluate whether the overall risk estimate would have been affected by the data of individual study. Publication bias was assessed by the application of Egger's linear regression test [16] and Begg's rank correlation test [17].

We used the method developed by Greenland and Longnecker and the available Stata code written by Orsini et al. to estimate dose-response association [18, 19]. We extracted data on intake of dietary calcium or vitamin $\mathrm{D}$ in each category (the range or median or mean intake), the number of cases and participants (or person-years) in each category, and the RR (or HR) with $95 \% \mathrm{CI}$. The midpoint of the lower and upper categories was regarded as median of the quantile, if the medians or means were not provided. If the highest category with an open upper boundary, we considered the width of that category was the same to the adjacent category. If the lowest category was open-ended, it was assumed to be zero. To evaluate possible non-linear associations between the intake of dietary calcium or vitamin $\mathrm{D}$ and the risk of diabetes, restricted cubic splines was used, with 3 knots at fixed percentiles of 10th, 50th, and 90th of the distribution [20]. All analyses were conducted with Stata 14.0 (StatCorp, College Station, TX, USA) and $P<0.05$ was considered statistically significant.

\section{Results}

\subsection{Search Results and Study Characteristics}

As the flow chart showed (Figure 1), a total of 9461 citations (excluding duplicates) from PubMed $(n=3539)$, Web of Science $(n=5961)$, and Embase $(n=5817)$ were initially identified. After reviewing titles and abstracts, we retrieved 130 full-text articles. Ultimately, 22 studies in 15 articles [21-35] were eligible for our inclusion in this meta-analysis. Our meta-analysis included a total of 448578 participants and 31027 cases of T2DM. The details of the 22 studies were 
presented in Table1. The average score of quality assessment was 7.3 (range from 5-9) (Table 3).

Table 1. Characteristics of the 15 articles included in the meta-analysis.

\begin{tabular}{|c|c|c|c|c|c|c|c|}
\hline Author, Year & Country & Gender & $\begin{array}{l}\text { Age Range } \\
\text { (Years) }\end{array}$ & $\begin{array}{l}\text { Participants } \\
\text { (Cases) }\end{array}$ & $\begin{array}{l}\text { Follow-up } \\
\text { (years) }\end{array}$ & Exposure & $\begin{array}{l}\text { Intake Comparison } \\
\text { (Highest vs. Lowest) }\end{array}$ \\
\hline Colditz, 1992 (18) & America & $\mathrm{F}$ & $34-59$ & $84360(702)$ & 6 & Dietary calcium & Q5 vs. Q1 \\
\hline Gagnon, 2011 (19) & Australia & Both & $\geq 25$ & $5200(199)$ & 5 & Dietary calcium & $\begin{array}{l}171-740 \mathrm{mg} / \mathrm{d} \\
v s .1060-2317 \mathrm{mg} / \mathrm{d}\end{array}$ \\
\hline Kim, 2018 (20) & Korea & Both & $40-69$ & 8574 (1173) & 10 & Dietary calcium & Q4 vs. Q1 \\
\hline Kirii, 2009 (21) & Japan & $\begin{array}{l}\mathrm{M} \\
\mathrm{F}\end{array}$ & $40-69$ & $\begin{array}{l}25877(634) \\
33919(480)\end{array}$ & 10 & $\begin{array}{l}\text { Dietary calcium } \\
\text { Dietary vitamin D }\end{array}$ & $\begin{array}{l}439 \mathrm{mg} / \mathrm{d} v \mathrm{~s} .348 \mathrm{mg} / \mathrm{d} \\
18.0 \mu \mathrm{g} / \mathrm{d} v s .4 .7 \mu \mathrm{g} / \mathrm{d}\end{array}$ \\
\hline Liu, 2005 (22) & America & $\mathrm{F}$ & $>45$ & $10066(805)$ & 8.8 & $\begin{array}{l}\text { Dietary calcium } \\
\text { Dietary vitamin D }\end{array}$ & $\begin{array}{l}1168 \mathrm{mg} / \mathrm{d} v s .486 \mathrm{mg} / \mathrm{d} \\
377 \mathrm{IU} / \mathrm{d} v s . \leq 111 \mathrm{IU} / \mathrm{d}\end{array}$ \\
\hline Oh, 2017 (23) & Korea & $\begin{array}{l}\mathrm{M} \\
\mathrm{F}\end{array}$ & $>40$ & $\begin{array}{l}2830(122) \\
4669(168)\end{array}$ & 6 & Dietary calcium & $462 \mathrm{mg} / \mathrm{d} v s .197 \mathrm{mg} / \mathrm{d}$ \\
\hline Pittas, 2006 (24) & America & $\mathrm{F}$ & $30-55$ & $\begin{array}{l}83779(2465) \\
79232(4547)\end{array}$ & 20 & $\begin{array}{l}\text { Dietary calcium } \\
\text { Dietary vitamin D }\end{array}$ & $\begin{array}{l}>1000 \mathrm{mg} / \mathrm{d} v s . \leq 500 \mathrm{mg} / \mathrm{d} \\
>400 \mathrm{IU} / \mathrm{d} v s . \leq 100 \mathrm{IU} / \mathrm{d}\end{array}$ \\
\hline Talaei, 2018 (25) & Singapore & Both & $45-74$ & $45411(5207)$ & 11 & Dietary calcium & $597 \mathrm{mg} / \mathrm{d} v s .258 \mathrm{mg} / \mathrm{d}$ \\
\hline van Dam, 2006 (26) & America & $\mathrm{F}$ & $21-69$ & $41186(1964)$ & 8 & Dietary calcium & $661 \mathrm{mg} / \mathrm{d} v s .219 \mathrm{mg} / \mathrm{d}$ \\
\hline Villegas, 2009 (27) & China & $\mathrm{F}$ & $40-70$ & $64191(2270)$ & 6.9 & Dietary calcium & $649.6 \mathrm{mg} / \mathrm{d} v s .277 .5 \mathrm{mg} / \mathrm{d}$ \\
\hline Wu, 2019 (28) & Finland & $\begin{array}{l}M \\
F\end{array}$ & $3-18$ & $\begin{array}{l}556(31) \\
578(19)\end{array}$ & 31 & Dietary calcium & $>800 \mathrm{mg} / \mathrm{d} v s . \leq 800 \mathrm{mg} / \mathrm{d}$ \\
\hline Yáñez, 2017 (29) & $\begin{array}{l}\text { Sweden, } \\
\text { America }\end{array}$ & $\mathrm{F}$ & $>18$ & $3414(169)$ & 12 & Dietary calcium & $\geq 1527 \mathrm{mg} / \mathrm{d} v s .<795 \mathrm{mg} / \mathrm{d}$ \\
\hline Abbas, 2014 (30) & Europe & Both & $46.5-59.2$ & 27043 (11994) & 10.8 & Dietary vitamin D & $\geq 6.07 \mu \mathrm{g} / \mathrm{d} v s .<2.19 \mu \mathrm{g} / \mathrm{d}$ \\
\hline Bao, 2018 (31) & America & $\mathrm{F}$ & $22-44$ & $8621(395)$ & 10 & Dietary vitamin D & $\geq 400 \mathrm{IU} / \mathrm{d} v s .<200 \mathrm{IU} / \mathrm{d}$ \\
\hline Eshak, 2018 (32) & Japan & Both & $40-79$ & $19168(494)$ & 5 & Dietary vitamin $\mathrm{D}$ & $13.3 \mu \mathrm{g} / \mathrm{d} v s .3 .7 \mu \mathrm{g} / \mathrm{d}$ \\
\hline
\end{tabular}

Table 1. Continued.

\begin{tabular}{|c|c|}
\hline Author, Year & Adjustment for Covariates \\
\hline Colditz, 1992 (18) & age, BMI, alcohol intake, family history of diabetes, prior weight change, time period, dietary magnesium, dietary potassium \\
\hline Gagnon, 2011 (19) & N/A \\
\hline Kim, $2018(20)$ & $\begin{array}{l}\text { age, sex, residential area, monthly family income, tobacco smoking, alcohol intake, physical activity, and BMI, systolic blood } \\
\text { pressure, diastolic blood pressure, and serum creatinine level, serum calcium intake }\end{array}$ \\
\hline Kirii, 2009 (21) & $\mathrm{N} / \mathrm{A}$ \\
\hline Liu, 2005 (22) & N/A \\
\hline Pittas, $2006(24)$ & $\begin{array}{l}\text { age, BMI, hypertension, family history of diabetes, smoking, physical activity, caffeine, alcohol, and state of residence, type of fat, } \\
\text { cereal fiber, glycemic load, magnesium, retinol, multivitamin use }\end{array}$ \\
\hline Talaei, $2018(25)$ & $\begin{array}{l}\text { age, sex, dialect, year of interview, and educational level, BMI, physical activity, smoking status, alcohol use, baseline history of } \\
\text { self-reported hypertension, and total energy intake, vegetable, fruit, soy-rich pattern and dim sum and meat-rich pattern, coffee, } \\
\text { and soda, dietary intake of potassium, magnesium, phosphorus, and vitamin D }\end{array}$ \\
\hline van Dam, 2006 (26) & $\begin{array}{l}\text { age, total energy intake, BMI, smoking status, strenuous physical activity, alcohol consumption, parental history of diabetes, } \\
\text { education level, coffee consumption, sugar-sweetened soft drink consumption, and quintiles of processed meat and other red meat } \\
\text { consumption, magnesium intake. }\end{array}$ \\
\hline Villegas, 2009 (27) & $\begin{array}{l}\text { age, energy intake, BMI, waist-hip ratio, smoking status, alcohol consumption, physical activity, income level, education level, } \\
\text { occupation, and hypertension. }\end{array}$ \\
\hline $\mathrm{Wu}, 2019(28)$ & $\begin{array}{l}\text { age, childhood and adulthood BMI, baseline serum } 25 \mathrm{OHD} \text { levels, parental history of diabetes, fruit and vegetable consumption, } \\
\text { physical activity, smoking, and socioeconomic status (parental education years) }\end{array}$ \\
\hline Yáñez, 2017 (29) & $\begin{array}{l}\text { daily energy intake, maternal age, race/ethnicity, educational attainment, cigarette smoking status, pre-pregnancy BMI, prenatal } \\
\text { vitamin use, physical activity, family history of diabetes, alcohol, coffee, sugar-sweetened beverages, red and processed meats, } \\
\text { fatty fish, total fiber intake, dietary magnesium and vitamin D intake }\end{array}$ \\
\hline Abbas, $2014(30)$ & $\begin{array}{l}\text { sex, age and study center, total energy, non-fat energy, polyunsaturated fatty acids, monounsaturated fatty acids, saturated fatty } \\
\text { acids, physical activity, education, BMI, smoking, alcohol intake }\end{array}$ \\
\hline Bao, $2018(31)$ & $\begin{array}{l}\text { age, parity, race/ethnicity, family history of diabetes, cigarette smoking, alcohol intake, physical activity, estimated ultraviolet B } \\
\text { flux, and BMI, total energy intake, intakes of saturated fat, monounsaturated fatty acids, polyunsaturated fatty acids, } \\
\text { transunsaturated fatty acids, dietary cholesterol, glycemic load, dietary fiber, magnesium, calcium, vitamin A. }\end{array}$ \\
\hline Eshak, 2018 (32) & N/A \\
\hline
\end{tabular}




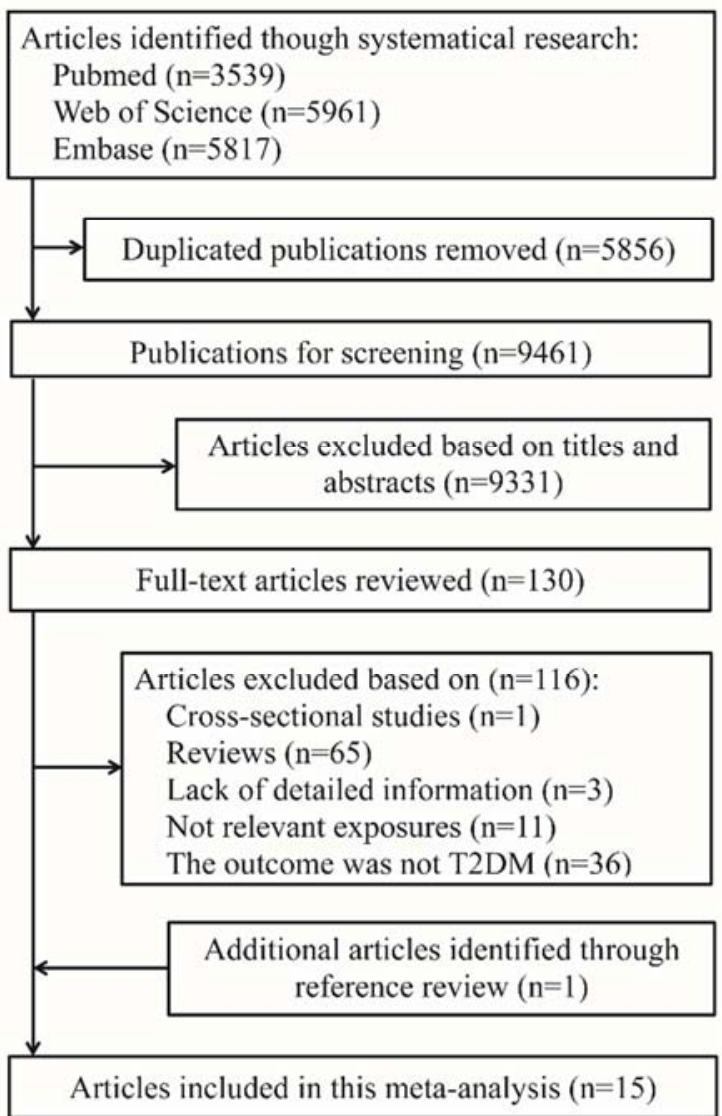

Figure 1. Flow diagram depicting the screening and exclusion of publications included in the meta-analysis.

\subsection{Dietary Calcium intake and T2DM}

The overall analysis revealed an inverse association between dietary intake of calcium and risk of T2DM based on the random-effects model (RR: 0.84 ; 95\% CI: 0.76-0.93; $I^{2}=54.7 \%$ ) (Figure $\left.2 \mathrm{~A}\right)$. Ten studies provided the required data for dose-response analysis and an inverse linear association between dietary calcium intake and T2DM risk was observed $(P=0.002)$. The dose-response analysis also suggested that each $300 \mathrm{mg} / \mathrm{d}$ dietary calcium intake increase reduced the risk of T2DM by $8 \%$ (RR: 0.92 ; $95 \%$ CI: $0.87-$ 0.97) (Figure 2B). However, no significant non-linear

A

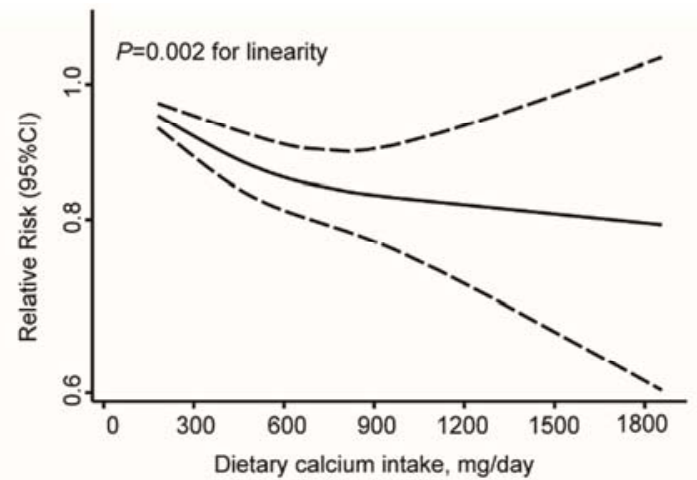

association was observed by the restricted cubic splines model $(\mathrm{P}=0.119$, Figure $3 \mathrm{~A})$.

\subsection{Dietary Vitamin D intake and T2DM}

Consistent with all independent studies included in our analyses, no significant association was found between dietary vitamin D intake and T2DM risk (RR: 1.00 ; 95\% CI: $0.92-1.08 ; \mathrm{I}^{2}=0.0 \%$ ) (Figure $\left.4 \mathrm{~A}\right)$. The dose-response analysis suggested that no significant association between dietary vitamin D intake and T2DM risk by the per $300 \mathrm{IU} /$ day increase (RR: 1.02, 95\% CI, $0.97-1.06 ; \quad \mathrm{I} 2=19.6 \%$ ) (Figure4B). No evidence of a non-linear association was found between dietary vitamin $\mathrm{D}$ intake and T2DM risk $(\mathrm{P}=0.959$ for non-linearity, Figure $3 \mathrm{~B})$.

A

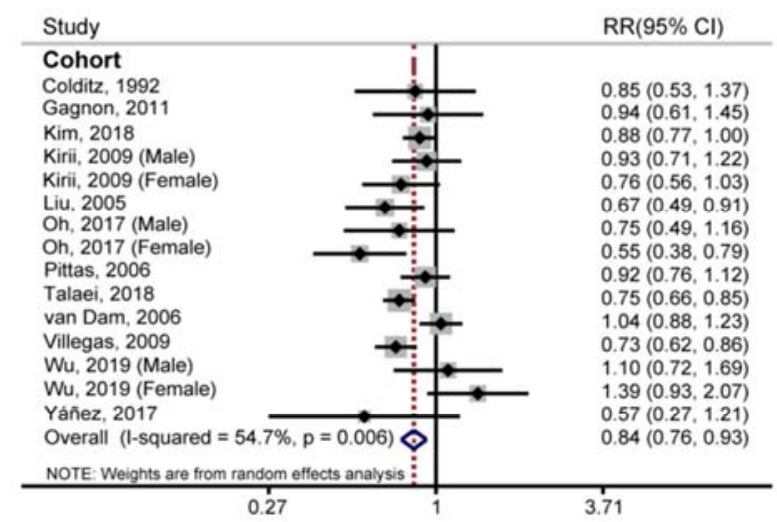

B

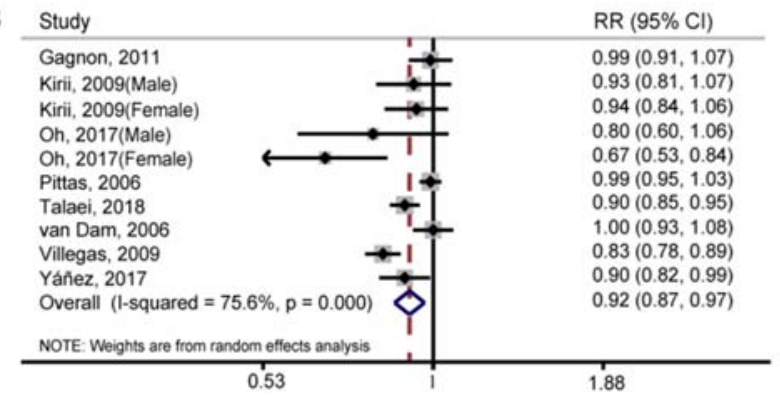

Figure 2. Forest plots summarizing the RR of T2DM for the highest vs. the lowest category of $(A)$ dietary calcium intake and (B) and per $300 \mathrm{mg} /$ day increase in dietary calcium intake. RR: relative risk; CI: confidence interval.

B

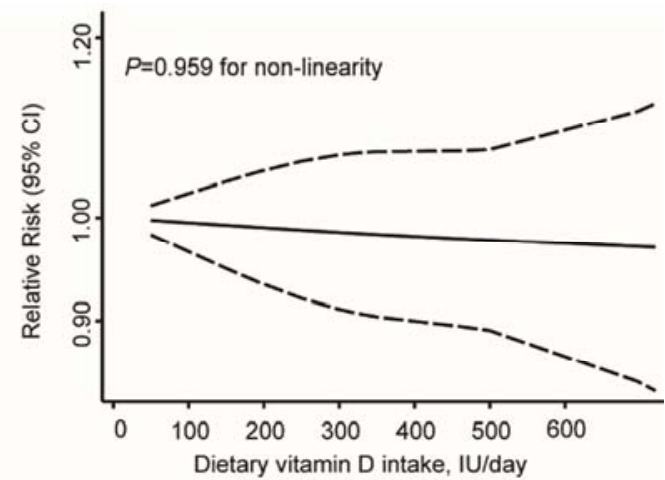

Figure 3. Dose-response analyses of the non-linear association between (A) dietary calcium; (B) dietary vitamin D intake and the risk of T2DM. In each panel, the solid line and dashed lines represent the estimated RR and $95 \%$ CI, respectively. 
A Dose-Response Meta-analysis

Table 2. Subgroup analyses between dietary calcium intake and the risk of T2DM.

\begin{tabular}{|c|c|c|c|c|c|}
\hline group & $\mathbf{N}$ & Cases/controls & RR $(95 \%$ CI) & $I^{2}$ & $\boldsymbol{P}$ \\
\hline \multicolumn{6}{|c|}{ Geographic Location } \\
\hline Asia & 7 & $9862 / 175835$ & $0.79(0.73,0.84)$ & $36.3 \%$ & 0.152 \\
\hline Europe & 3 & $249 / 6085$ & $1.14(0.90,1.45)$ & $0.0 \%$ & 0.418 \\
\hline America & 5 & $8033 / 193682$ & $0.92(0.82,1.03)$ & $48.5 \%$ & 0.101 \\
\hline \multicolumn{6}{|l|}{ Gender } \\
\hline Men & 3 & $787 / 28476$ & $0.92(0.75,1.13)$ & $0.0 \%$ & 0.466 \\
\hline Women & 9 & $10970 / 294102$ & $0.82(0.70,0.97)$ & $67.6 \%$ & 0.002 \\
\hline \multicolumn{6}{|l|}{ Mean age } \\
\hline$\leq 50$ & 6 & $7278 / 185505$ & $1.00(0.90,1.12)$ & $21.3 \%$ & 0.273 \\
\hline$>50$ & 9 & $10866 / 190097$ & $0.78(0.73,0.84)$ & $27.8 \%$ & 0.197 \\
\hline \multicolumn{6}{|c|}{ Follow-up years } \\
\hline$\geq 10$ & 8 & $12364 / 189970$ & $0.85(0.79,0.92)$ & $49.9 \%$ & 0.051 \\
\hline$<10$ & 7 & $5780 / 185632$ & $0.78(0.65,0.94)$ & $63.5 \%$ & 0.012 \\
\hline \multicolumn{6}{|l|}{ Participants } \\
\hline Youth & 2 & $50 / 1084$ & $1.25(0.93,1.67)$ & $0.0 \%$ & 0.432 \\
\hline Pregnancy & 1 & $169 / 3245$ & $0.57(0.27,1.21)$ & - & - \\
\hline Healthy adult & 12 & $17925 / 371273$ & $0.83(0.78,0.88)$ & $49.7 \%$ & 0.025 \\
\hline \multicolumn{6}{|c|}{ Adjustment for alcohol } \\
\hline Yes & 7 & $15686 / 294365$ & $0.84(0.75,0.95)$ & $58.7 \%$ & 0.024 \\
\hline No & 8 & $2458 / 81237$ & $0.84(0.69,1.02)$ & $57.2 \%$ & 0.022 \\
\hline \multicolumn{6}{|c|}{ Adjustment for magnesium } \\
\hline Yes & 7 & $12725 / 231834$ & $0.81(0.68,0.96)$ & $63.8 \%$ & 0.011 \\
\hline No & 8 & $5419 / 143768$ & $0.86(0.75,0.99)$ & $50.9 \%$ & 0.047 \\
\hline \multicolumn{6}{|c|}{ Adjustment for energy intake } \\
\hline Yes & 4 & $9610 / 144592$ & $0.81(0.66,0.99)$ & $75.8 \%$ & 0.006 \\
\hline No & 11 & $8534 / 231010$ & $0.87(0.80,0.94)$ & $41.7 \%$ & 0.071 \\
\hline \multicolumn{6}{|c|}{ Adjustment for vitamin D intake } \\
\hline Yes & 2 & $5376 / 43449$ & $0.74(0.66,0.84)$ & $0.0 \%$ & 0.479 \\
\hline No & 13 & $12768 / 332153$ & $0.86(0.77,0.96)$ & $53.8 \%$ & 0.011 \\
\hline \multicolumn{6}{|c|}{ Adjustment for age } \\
\hline Yes & 11 & $16026 / 302658$ & $0.85(0.75,0.96)$ & $63.8 \%$ & 0.002 \\
\hline No & 4 & $2118 / 72944$ & $0.81(0.69,0.95)$ & $2.2 \%$ & 0.381 \\
\hline
\end{tabular}

A

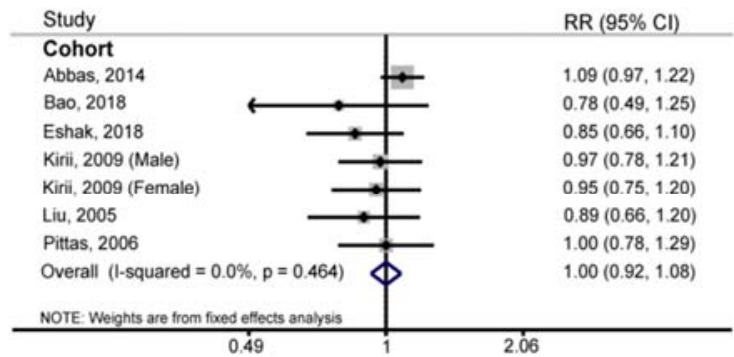

B

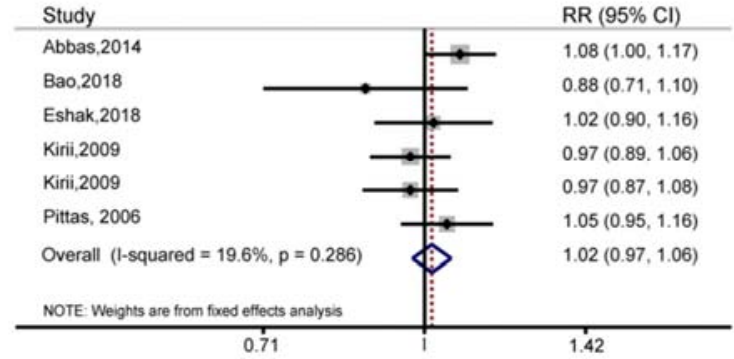

Figure 4. Forest plots summarizing the RR of T2DM for the highest vs. the lowest category of (A) dietary vitamin D intake and (B) and per 300 IU/day increase in dietary vitamin $D$ intake. RR: relative risk; $C I$ : confidence interval.

\subsection{Heterogeneity and Meta-regression}

As for dietary vitamin D intake, there is low study heterogeneity. In contrast, moderate heterogeneity was found in the analysis of dietary calcium intake $\left(I^{2}=54.7 \%, P=0.006\right)$ (Figure 2). Univariate meta-regression analysis on dietary calcium, including covariates of location $(P=0.989)$, gender $(P=0.936)$, mean age $(P=0.106)$, intake of alcohol $(P=0.349)$, dietary magnesium $(P=0.149)$, vitamin $\mathrm{D}(P=0.123)$, and energy $(P=0.214)$, did not show that any covariation had a significant impact on between-study heterogeneity.

\subsection{Subgroup Analyses Stratified by Possible Confounders}

Next, we a subgroup analysis was performed on dietary calcium to test the stability of results based on geographic location and gender. The analysis revealed an inverse association in Asia but not in Europe and America, and in women, but not in men. When stratifying studies by mean age in the study, an inverse association was found in the subgroup of ages older than 50 years old, but not in the subgroup of ages younger and equal to 50 (Table 2). In addition, the significant association persisted even after adjusting for major confounding factors, such as intake of dietary 
magnesium, vitamin D, total energy, and alcohol consumption (Table 2).

were consistent with the overall results of dietary source (Table 4).

As for dietary vitamin $\mathrm{D}$, results of our subgroup analyses

Table 3. Quality assessment of all included studies.

\begin{tabular}{lllll}
\hline \multirow{2}{*}{ Author, year } & Selection & & & \\
\cline { 2 - 5 } & Representativeness of cases & Selection of controls & Ascertainment of exposure & No history of outcome \\
\hline Colditz, 1992 (1) & 0 & 1 & 1 & 1 \\
Gagnon, 2011 (2) & 0 & 1 & 0 & 1 \\
Kim, 2018 (3) & 1 & 1 & 1 & 1 \\
Kirii, 2009 (4) & 0 & 1 & 1 & 1 \\
Liu, 2005(5) & 0 & 1 & 1 & 1 \\
Oh, 2017 (6) & 0 & 1 & 1 & 1 \\
Pittas, 2006 (7) & 0 & 1 & 1 & 1 \\
Talaei, 2018 (8) & 0 & 1 & 1 & 1 \\
Van Dam, 2006 (9) & 0 & 1 & 1 & 1 \\
Villegas, 2009 (10) & 0 & 1 & 1 & 1 \\
Wu, 2019(11) & 0 & 1 & 1 & 1 \\
Yáñez, 2017 (12) & 0 & 1 & 1 & 1 \\
Abbas, 2014 (13) & 1 & 1 & 1 & 1 \\
Bao, 2018 (14) & 0 & 1 & 1 & 1 \\
Eshak, 2018(15) & 1 & & 1 & \\
Average Score: 7.3 & & & & 1 \\
\hline
\end{tabular}

Table 3. Continued.

\begin{tabular}{|c|c|c|c|c|c|}
\hline \multirow{2}{*}{ Author, year } & \multirow{2}{*}{$\begin{array}{l}\text { Comparability } \\
\text { Comparable On confounders }\end{array}$} & \multicolumn{3}{|l|}{ Outcome } & \multirow{2}{*}{$\begin{array}{l}\text { Total } \\
\text { Score }\end{array}$} \\
\hline & & Outcome assessment & Adequate follow-uptime ( $\geq 5$ years) & Follow-uprate (>80\%) & \\
\hline Colditz, 1992 (1) & 2 & 1 & 1 & 1 & 8 \\
\hline Gagnon, 2011 (2) & 0 & 1 & 1 & 1 & 5 \\
\hline Kim, 2018 (3) & 2 & 1 & 1 & 1 & 9 \\
\hline Kirii, 2009 (4) & 0 & 0 & 1 & 1 & 5 \\
\hline Liu, 2005 (5) & 0 & 1 & 1 & 1 & 6 \\
\hline Oh, $2017(6)$ & 1 & 1 & 1 & 1 & 7 \\
\hline Talaei, 2018 (8) & 2 & 1 & 1 & 1 & 8 \\
\hline Van Dam, 2006 (9) & 2 & 1 & 1 & 1 & 8 \\
\hline Villegas, 2009 (10) & 1 & 1 & 1 & 1 & 7 \\
\hline Wu, 2019 (11) & 2 & 1 & 1 & 1 & 8 \\
\hline Yáñez, 2017 (12) & 2 & 1 & 1 & 1 & 8 \\
\hline Abbas, 2014 (13) & 1 & 1 & 1 & 1 & 8 \\
\hline Bao, 2018 (14) & 2 & 1 & 1 & 1 & 8 \\
\hline
\end{tabular}

Table 4. Subgroup analyses between dietary vitamin D intake and the risk of T2DM.

\begin{tabular}{|c|c|c|c|c|c|}
\hline Subgroup & $\mathbf{N}$ & Cases/controls & $\operatorname{RR}(95 \% C I)$ & $I^{2}$ & $\boldsymbol{P}$ \\
\hline Geographic Location & & & & & \\
\hline Asia & 3 & $1608 / 77356$ & $0.93(0.81,1.06)$ & 0.0 & 0.732 \\
\hline Europe & 1 & $11994 / 15049$ & $1.09(0.97,1.22)$ & - & - \\
\hline $\begin{array}{l}\text { America } \\
\text { Gender }\end{array}$ & 3 & $5747 / 96719$ & $0.93(0.78,1.11)$ & $0.0 \%$ & 0.626 \\
\hline Male & 1 & $634 / 25243$ & $0.97(0.78,1.21)$ & - & - \\
\hline $\begin{array}{l}\text { Female } \\
\text { Adjustment for calcium intake }\end{array}$ & 4 & $6227 / 130158$ & $0.93(0.81,1.08)$ & $0.0 \%$ & 0.810 \\
\hline yes & 1 & $395 / 8226$ & $0.78(0.49,1.26)$ & & \\
\hline $\begin{array}{l}\text { no } \\
\text { Follow-up years }\end{array}$ & 6 & $18954 / 180898$ & $1.01(0.93,1.09)$ & $0.0 \%$ & 0.473 \\
\hline$\geq 10$ & 5 & $18050 / 161189$ & $1.03(0.94,1.12)$ & $0.0 \%$ & 0.547 \\
\hline$<10$ & 2 & $1299 / 27935$ & $0.87(0.71,1.05)$ & $0.0 \%$ & 0.817 \\
\hline Participants & & & & & \\
\hline Pregnancy & 1 & $395 / 8226$ & $0.78(0.49,1.26)$ & & \\
\hline yes & 3 & $16936 / 102507$ & $1.06(0.96,1.17)$ & $2.6 \%$ & 0.358 \\
\hline no & 4 & $2413 / 86617$ & $1.06(0.96,1.17)$ & $0.0 \%$ & 0.870 \\
\hline Adjustment for magnesium & & & & & \\
\hline
\end{tabular}




\begin{tabular}{|c|c|c|c|c|c|}
\hline Subgroup & $\mathbf{N}$ & Cases/controls & RR $(95 \% C I)$ & $I^{2}$ & $P$ \\
\hline yes & 2 & $4942 / 87458$ & $0.95(0.76,1.18)$ & $0.0 \%$ & 0.363 \\
\hline no & 5 & $14407 / 101666$ & $1.01(0.93,1.10)$ & $12.0 \%$ & 0.337 \\
\hline \multicolumn{6}{|c|}{ Adjustment for energy intake } \\
\hline yes & 2 & $12389 / 23275$ & $1.07(0.96,1.20)$ & $45.1 \%$ & 0.177 \\
\hline no & 5 & $6960 / 165849$ & $0.94(0.84,1.05)$ & $0.0 \%$ & 0.903 \\
\hline \multicolumn{6}{|c|}{ Adjustment for age } \\
\hline yes & 3 & $16936 / 102507$ & $1.06(0.96,1.17)$ & $2.6 \%$ & 0.358 \\
\hline no & 4 & $2413 / 86617$ & $0.92(0.81,1.04)$ & $0.0 \%$ & 0.870 \\
\hline
\end{tabular}

\subsection{Publication Bias and Sensitivity Analysis}

Our sensitivity analysis indicated that there was no significant change when any one study was omitted at a time for dietary calcium and vitamin D (Figure 5). We then looked for publication bias using Begg's funnel plots and Egger's test. As for dietary calcium, result revealed no significant publication bias based on Egger's test $(P=0.966)$ (Figure 6A). With respect to dietary vitamin $\mathrm{D}$, publication bias was statistically significant $(P=0.003)$ (Figure 6B). We found that result displayed no significant publication bias $(P=0.113)$ after excluding the study performed by Abbas et al. (30), which used 24-h diet recall data, and most probably, such an exposure assessment might not be accepted for publication today.

A

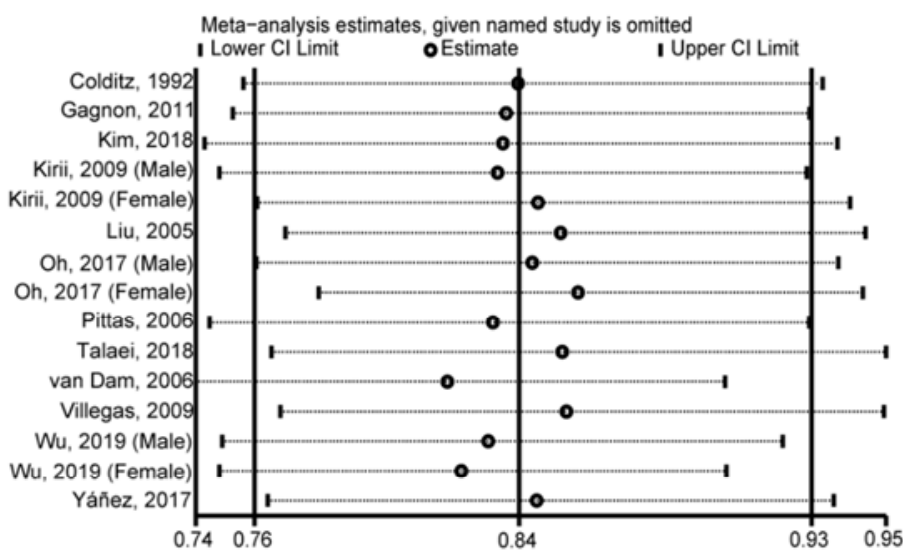

B

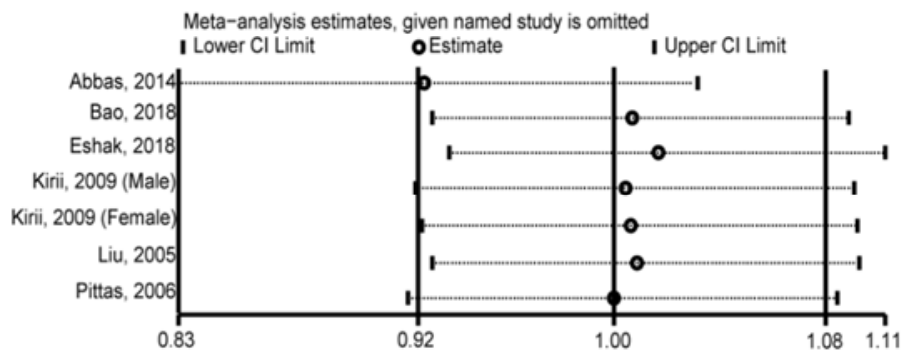

Figure 5. Analysis of influence of individual study on the association between (A) dietary calcium; (B) dietary vitamin D and the risk of T2DM. Open circle indicates the pooled RR, given named study is omitted. Horizontal lines represent the $95 \%$ CI.

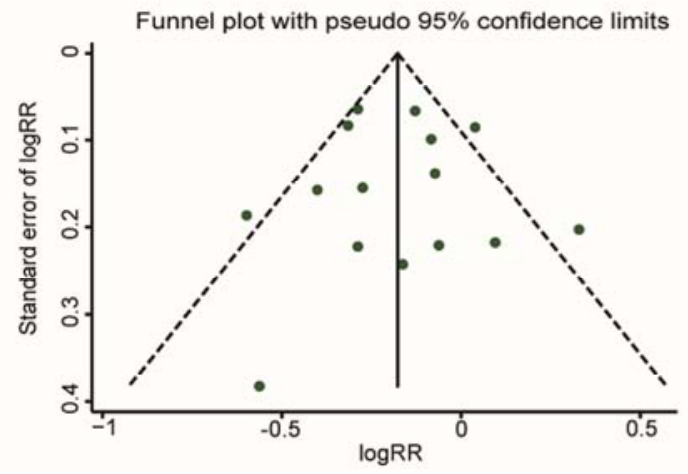

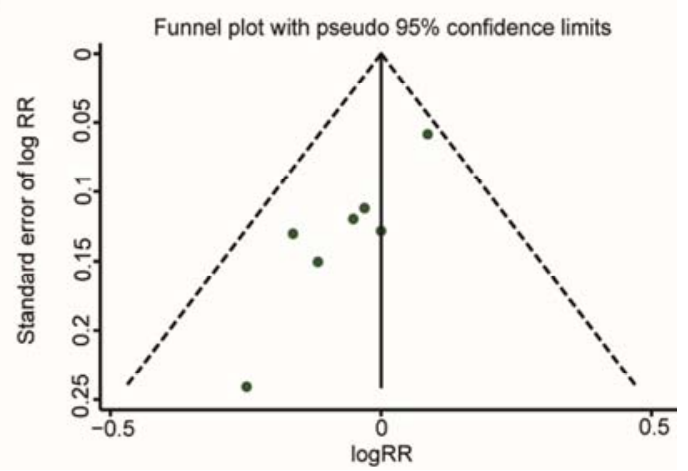

Figure 6. Funnel plot of $(A)$ dietary calcium; $(B)$ dietary vitamin $D$ and the risk of T2DM. 


\section{Discussion}

The meta-analysis presented in this paper was based on 22 published studies including 448578 participants and 31027 cases of T2DM. We found an inverse association between T2DM risk and dietary calcium intake. Moreover, our dose-response analysis revealed that each $300 \mathrm{mg} /$ day increase in dietary calcium intake reduces T2DM risk by $8 \%$. On the other hand, we found no significant association between dietary vitamin $\mathrm{D}$ intake and T2DM risk.

As to the correlation between dietary calcium intake and T2DM risk, a previous meta-analysis concluded that dietary calcium intake was not independently associated with T2DM risk and that the inverse association between dietary calcium intake and T2DM risk in prior observational studies may be influenced by magnesium intake [9]. However, in our analysis, after adjusted for dietary magnesium, an inverse correlation between dietary calcium intake and T2DM risk still exists. The reason for the different results may be that our meta-analysis included more studies (15 vs. 7) and incident cases (18144 vs. 11195 ) than the previous meta-analysis. Nevertheless, we found that inverse relationship was only observed in Asia, but not in America and Europe. Racial differences in calcium metabolism may explain the result, for example, dietary calcium is likely utilized more efficiently in Chinese than Westerners as reported [36]. Moreover, a subgroup analysis revealed that this inverse correlation was present in women but not in men. The gender disparity could be ascribed to sex hormones. However, most women in our study were post-menopausal, and the inverse association also exists in subgroup of age above 50, possibly there were other explanations related to lifestyle. Women were inclined to consumed more calcium and dairy products than men [24]. On the other hand, smoking and alcohol consumption, which were common among males, may influence calcium metabolism via decline of the vitamin D parathyroid hormone system among smokers [37] or by reduction of bone mineral density in alcohol drinkers [38]. Additionally, post-menopausal women were calcium deficiency [39]. In our subgroup analyses, alcohol was identified as a confounder. As only two studies were adjusted for vitamin D intake, it was unclear whether the protective effect of dietary calcium was independent or depending on vitamin D.

Different from dietary calcium, our analysis suggests that there is no association between dietary vitamin $\mathrm{D}$ intake and T2DM risk. It is reported that vitamin D can affect glucose homeostasis such as insulin secretion, insulin sensitivity and systemic inflammation [8]. Animal studies and human observational studies linked vitamin D deficiency to numerous chronic diseases including hypertension, and diabetes [7, 40]. However, multiple RCTs have failed to demonstrate significant benefits of vitamin D supplementation for diabetes. It is possible that many prior epidemiological associations between vitamin D deficiency and chronic diseases were caused by unmeasured residual confounding. For many of the chronic diseases previously relation to vitamin D deficiency, their preclinical stages likely lead to the vitamin D deficiency that subsequently cause false associations in some observational studies [41], so our results demonstrate that dietary vitamin D may have no protective effect on T2DM in people with normal vitamin D levels at baseline.

Our meta-analysis has several strengths. First, this is the first systemic dose-response meta-analysis that quantifies the association between the intake of dietary calcium or vitamin $\mathrm{D}$ and T2DM risk. Second, our meta-analysis included prospective studies from large populations and long-term follow-up studies, and thus increases the statistical power by detecting modest associations. Third, we performed several subgroup analyses to identify the potential sources of heterogeneity and to evaluate the robustness of our results. We found difference between subgroups of mean ages, geographical areas and gender-specific difference in the effects of dietary calcium intake on the risk of T2DM. Lastly, we included the studies which used estimates from the fully adjusted models to minimize the effects of potential confounding factors.

Our analysis has limitations. First, the possibility of residual confounding still exists, given the observational nature of the studies. Second. inaccurate measurement seemed inevitable and might weaken true associations, because the consumption of various dietary factors was assessed by food frequency questionnaires in almost all of cohort studies. Third, a number of included studies did not adjust the vitamin D information when assessing the relationship between dietary calcium intake and the outcome. It is also not clear whether the protective effect of dietary calcium on T2DM is independent or not. Finally, there was some evidence of publication bias as for dietary vitamin D. We cannot completely rule out the possibility that it affected the significance of our results.

Large-scale epidemiological studies, in particular prospective cohort studies that adjust for vitamin $\mathrm{D}$, calcium intake or other possible covariates separately should be conducted in order to further test the association between dietary calcium or vitamin D intake and the risk of T2DM. Additionally, researches are needed to examine the effects of dietary calcium and/or vitamin $\mathrm{D}$ intake on indexes such as glucose tolerance, insulin secretion, etc. to improve homeostasis rates of glucose and insulin among non-diabetic subjects.

\section{Conclusions}

Our meta-analysis indicates that dietary calcium intake is inversely correlated with T2DM risk and dose-response analysis suggests that for each $300 \mathrm{mg} / \mathrm{d}$ increase in dietary calcium intake reduces the risk of T2DM by $8 \%$. The inverse association is only found in Asia but not Europe and America, and in women, but not in men. In contrast, we found no correlation between dietary vitamin $\mathrm{D}$ intake and the risk of T2DM. Our results strongly support the general notion that sufficient calcium are protective factors for T2DM incidence. 
The dose-response analyses also consist with these findings. These results may have important public health implications in the prevention of T2DM incidence.

\section{Availability of Data and Materials}

All data generated or analyzed during the study are included in this published article.

\section{Authors' Contributions}

L.L. and Q.L. designed the study; L.L. and Q.L. assessed the studies for inclusion, extracted the data, and assessed the quality of the included studies; L.L. and Q.L. conducted the meta-analysis; other authors tabulated the data and checked the details of all the files; L.L. wrote the first draft of the manuscript; and J.W. and H.W. provided critical input for the manuscript. Y.X., X.F., S.Z. and M.Z. participated in the helpful discussion and manuscript revision. Y.C. participated in the English grammar revision of the manuscript. W.Y. did critical revision of the manuscript for important intellectual content and study supervision. All authors have contributed significantly, and all authors are in agreement with respect to the content of the manuscript.

\section{Conflict of Interest}

The authors declare that they have no competing interests.

\section{References}

[1] Federation ID. IDF diabetes atlas 9th edition 2019, https://www.diabetesatlas.org/en/sections/worldwide-toll-of-di abetes.html; 2019 [accessed 10 December 2019].

[2] Lee DH, Yang M, Giovannucci EL, Sun Q, Chavarro JE. Mushroom consumption, biomarkers, and risk of cardiovascular disease and type 2 diabetes: a prospective cohort study of US women and men. AmJ Clin Nutr. 2019; 110 (3): 666-74.

[3] Wang X, Wu W, Zheng W, Fang X, Chen L, Rink L, et al. Zinc supplementation improves glycemic control for diabetes prevention and management: a systematic review and meta-analysis of randomized controlled trials. Am J Clin Nutr. 2019; 110 (1): 76-90.

[4] Pozzan T, Rizzuto R, Volpe P, Meldolesi J. Molecular and cellular physiology of intracellular calcium stores. Physiol Rev. 1994; 74 (3): 595-636.

[5] Milner RD, Hales CN. The role of calcium and magnesium in insulin secretion from rabbit pancreas studied in vitro. Diabetologia. 1967; 3 (1): 47-9.

[6] Wright DC, Hucker KA, Holloszy JO, Han DH. Ca2+ and AMPK both mediate stimulation of glucose transport by muscle contractions. Diabetes. 2004; 53 (2): 330-5.

[7] Holick MF. Vitamin D deficiency. N Engl J Med. 2007; 357 (3): 266-81.
[8] Berridge MJ. Vitamin D deficiency and diabetes. Biochem J. 2017; 474 (8): 1321-32.

[9] Dong JY, Qin LQ. Dietary calcium intake and risk of type 2 diabetes: possible confounding by magnesium. Eur J Clin Nutr. 2012; 66 (3): 408-10.

[10] Zhao LM, Tian XQ, Ge JP, Xu YC. Vitamin D intake and type 2 diabetes risk: a meta-analysis of prospective cohort studies. Afr Health Sci. 2013; 13 (4): 1130-8.

[11] Stroup DF, Berlin JA, Morton SC, Olkin I, Williamson GD, Rennie D, et al. Meta-analysis of observational studies in epidemiology: a proposal for reporting. Meta-analysis Of Observational Studies in Epidemiology (MOOSE) group. Jama. 2000; 283 (15): 2008-12.

[12] Wells GA, Shea B, O'Connell D, Peterson J, Welch V, Losos M, et al. The Newcastle-Ottawa Scale (NOS) for Assessing the Quality of Nonrandomised Studies in Meta-Analyses, http://www.ohri.ca/programs/clinical_epidemiology/oxford.as p; 1999 [accessed 13 December 2019].

[13] Higgins JP, Altman DG, Gøtzsche PC, Jüni P, Moher D, Oxman $\mathrm{AD}$, et al. The Cochrane Collaboration's tool for assessing risk of bias in randomised trials. BMJ (Clinical research ed) 2011; 343: d5928.

[14] Fang X, Wei J, He X, An P, Wang H, Jiang L, et al. Landscape of dietary factors associated with risk of gastric cancer: A systematic review and dose-response meta-analysis of prospective cohort studies. Eur J Cancer. 2015; 51 (18): 2820-32.

[15] Higgins JP, Thompson SG. Controlling the risk of spurious findings from meta-regression. Stat Med. 2004; 23 (11): 1663-82.

[16] Egger M, Davey Smith G, Schneider M, Minder C. Bias in meta-analysis detected by a simple, graphical test. BMJ (Clinical research ed) 1997; 315 (7109): 629-34.

[17] Begg CB, Mazumdar M. Operating characteristics of a rank correlation test for publication bias. Biometrics1994; 50 (4): 1088-101.

[18] Greenland S, Longnecker MP. Methods for trend estimation from summarized dose-response data, with applications to meta-analysis. Am J Epidemiol. 1992; 135 (11): 1301-9.

[19] Orsini N, Bellocco R, Greenland S. Generalized least squares for trend estimation of summarized dose-response data. Stata J. 2006; 6: 40-57.

[20] Harrell FE, Jr., Lee KL, Pollock BG. Regression models in clinical studies: determining relationships between predictors and response. J Natl Cancer Inst. 1988; 80 (15): 1198-202.

[21] Colditz GA, Manson JE, Stampfer MJ, Rosner B, Willett WC, Speizer FE: Diet and risk of clinical diabetes in women. Am J Clin Nutr. 1992; 55 (5): 1018-23.

[22] Gagnon C, Lu ZX, Magliano DJ, Dunstan DW, Shaw JE, Zimmet PZ, et al. Serum 25-hydroxyvitamin D, calcium intake, and risk of type 2 diabetes after 5 years: results from a national, population-based prospective study (the Australian Diabetes, Obesity and Lifestyle study). Diabetes care. 2011; 34 (5): 1133-8. 
[23] Kim KN, Oh SY, Hong YC. Associations of serum calcium levels and dietary calcium intake with incident type 2 diabetes over 10years: the Korean Genome and Epidemiology Study (KoGES). Diabetol Metab Syndr. 2018; 10: 50.

[24] Kirii K, Mizoue T, Iso H, Takahashi Y, Kato M, Inoue M, et al.Calcium, vitamin D and dairy intake in relation to type 2 diabetes risk in a Japanese cohort. Diabetologia. 2009; 52 (12): 2542-50.

[25] Liu S, Song Y, Ford ES, Manson JE, Buring JE, Ridker PM. Dietary calcium, vitamin $\mathrm{D}$, and the prevalence of metabolic syndrome in middle-aged and older U.S. women. Diabetes care 2005; 28 (12): 2926-32.

[26] Oh JM, Woo HW, Kim MK, Lee Y-H, Shin DH, Shin M-H, et al Dietary total, animal, vegetable calcium and type 2 diabetes incidence among Korean adults: The Korean Multi-Rural Communities Cohort (MRCohort). Nutr Metab Cardiovasc Dis 2017; 27 (12): 1152-64.

[27] Pittas AG, Dawson-Hughes B, Li T, Van Dam RM, Willett WC, Manson JE, et al. Vitamin D and calcium intake in relation to type 2 diabetes in women. Diabetes care 2006; 29 (3): 650-6.

[28] Talaei M, Pan A, Yuan JM, Koh WP: Dairy intake and risk of type 2 diabetes. Clin Nutr. 2018; 37 (2): 712-8.

[29] van Dam RM, Hu FB, Rosenberg L, Krishnan S, Palmer JR. Dietary calcium and magnesium, major food sources, and risk of type 2 diabetes in U.S. black women. Diabetes care 2006; 29 (10): 2238-43.

[30] Villegas R, Gao YT, Dai Q, Yang G, Cai H, Li H, et al. Dietary calcium and magnesium intakes and the risk of type 2 diabetes: the Shanghai Women's Health Study. Am J Clin Nutr. 2009; 89 (4): $1059-67$.

[31] Wu F, Juonala M, Pahkala K, Buscot M, Sabin MA, Pitkänen N, et al. Youth and Long-Term Dietary Calcium Intake With Risk of Impaired Glucose Metabolism and Type 2 Diabetes in Adulthood. J Clin Endocrinol Metab. 2019; 104 (6): 2067-74.
[32] Osorio-Yáñez C, Qiu C, Gelaye B, Enquobahrie DA, Williams MA. Risk of gestational diabetes mellitus in relation to maternal dietary calcium intake. Public Health Nutr. 2017; 20 (6): 1082-9.

[33] Abbas S, Linseisen J, Rohrmann S, Beulens JWJ, Buijsse B, Amiano $\mathrm{P}$, et al. Dietary vitamin D intake and risk of type 2 diabetes in the European Prospective Investigation into Cancer and Nutrition: the EPIC-InterAct study. Eur J Clin Nutr. 2014; 68 (2): 196-202.

[34] Bao W, Song Y, Bertrand KA, Tobias DK, Olsen SF, Chavarro JE, et al. Prepregnancy habitual intake of vitamin D from diet and supplements in relation to risk of gestational diabetes mellitus: A prospective cohort study. J Diabetes. 2018; 10 (5): 373-9.

[35] Eshak ES, Iso H, Muraki I, Tamakoshi A. Fat-soluble vitamins from diet in relation to risk of type 2 diabetes mellitus in Japanese population. Br J Nutr. 2018: 1-18.

[36] Fang AP, Li KJ, Shi HY, He JJ, Li H. Habitual dietary calcium intakes and calcium metabolism in healthy adults Chinese: a systematic review and meta-analysis. Asia Pac J Clin Nutr. 2016; 25 (4): 776-84.

[37] Brot C, Jorgensen NR, Sorensen OH. The influence of smoking on vitamin D status and calcium metabolism. Eur J Clin Nutr. 1999; 53 (12): 920-6.

[38] Conde FA, Aronson WJ. Risk factors for male osteoporosis. Urol Oncol. 2003; 21 (5): 380-3.

[39] Resnick NM, Greenspan SL. 'Senile' osteoporosis reconsidered. Jama. 1989; 261 (7): 1025-9.

[40] Gunta SS, Thadhani RI, Mak RH. The effect ofvitamin D status on risk factors for cardiovasculardisease. Nat Rev Nephrol. 2013; 9 (6): 337-47.

[41] LucasA, WolfM. Vitamin D and Health Outcomes: Then Came the Randomized Clinical Trials. Jama. 2019. 